\title{
Estimativa da Composição Corporal e Análise de Concordância Entre Analisadores de Impedância Bioelétrica Bipolar e Tetrapolar
}

\author{
Body Composition Estimation and Agreement Analysis Between \\ Bipolar and Tetrapolar Bioelectrical Impedance Analyzers
}

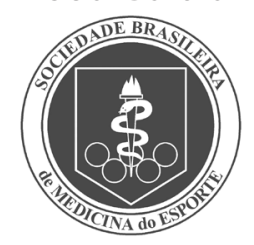

Artigo Original

\begin{abstract}
José Claudio Jambassi Filho1,2
Edilson Serpeloni Cyrino²

André Luiz Demantova Gurjão 1,2

Igor Augusto Braz²

Raquel Gonçalves'

Sebastião Gobbi'

1. Unesp - Universidade Estadual Paulista - IB - DEF - Laboratório de Atividade Física e Envelhecimento (Lafe) - Rio Claro/SP - Brasil. 2. Grupo de Estudo e Pesquisa em Metabolismo, Nutrição e Exercício. Centro de Educação Física e Esporte. Universidade Estadual de Londrina.
\end{abstract}

\section{Endereço para correspondência:}

Rua 5, 3.700, bloco 3, apto. 3 -

Jardim Portugal

13504-114 - Rio Claro, SP

E-mail: jambassifilho@yahoo.com.br

\begin{abstract}
RESUMO
O objetivo deste estudo foi analisar as estimativas da composição corporal e a concordância das medidas produzidas por meio de dois diferentes analisadores de impedância bioelétrica em homens adultos jovens. Vinte e dois sujeitos $\left(23,6 \pm 2,9\right.$ anos; $\left.73 \pm 6,8 \mathrm{~kg} ; 177,6 \pm 6,0 \mathrm{~cm} ; 23,1 \pm 1,9 \mathrm{~kg} / \mathrm{m}^{2}\right)$, aparentemente saudáveis, foram submetidos a medidas de impedância bioelétrica por meio dos analisadores tetrapolar de corpo inteiro (TCl, Biodynamic Body Composition Analyser, modelo 310) e bipolar para membros superiores (BMS, Omron ${ }^{\text {tm }}$, modelo HBF-306BL) após jejum de 12h. Estimativas da gordura corporal relativa (\%GC), massa livre de gordura (MLG) e massa gorda (MG) foram estabelecidas a partir das informações fornecidas pelos dois equipamentos. Nenhuma diferença estatisticamente significante foi verificada para \%GC $(10,9 \pm 4,3$ vs. $10,0 \pm 4,2 \% ; P=0,09)$, MG $(8,1 \pm 3,5$ vs. $7,4 \pm 3,5 \mathrm{~kg} ; P=0,10)$ e MLG $(64,9 \pm 5,2$ vs. 65,5 $\pm 4,9 \mathrm{~kg} ; P=0,09)$ entre TCl e BMS, respectivamente. Correlações positivas e significantes $(P<0,001)$ foram encontradas entre os analisadores para MLG $(r$ $=0,948), M G(r=0,891)$ e \%GC $(r=0,859)$. Os limites de concordância foram de $-0,6 \pm 3,2 \mathrm{~kg}$ para a MLG $(r=$ $0,135 ; P=0,551), 0,8 \pm 4,5 \%$ para a $\% G C(r=0,050 ; P=0,824)$ e $0,6 \pm 3,2 \mathrm{~kg}$ para a $M G(r=0,032 ; P=0,886)$. Os resultados sugerem que as estimativas da composição corporal por impedância bioelétrica não parecem ser afetadas pelo uso de diferentes analisadores (BMS vs. TCI) ou pela adiposidade corporal total.
\end{abstract}

Palavras-chave: massa livre de gordura, gordura corporal, impedância bioelétrica.

\begin{abstract}
The aim of this study was to analyze the body composition estimation and agreement of the measurements produced from two bioelectrical impedance analysers in young adult men. Twenty-two apparently healthy subjects $\left(23.6 \pm 2.9 \mathrm{yrs} ; 73.0 \pm 6.8 \mathrm{~kg} ; 177.6 \pm 6.0 \mathrm{~cm} ; 23.1 \pm 1.9 \mathrm{~kg} / \mathrm{m}^{2}\right)$, were submitted to bioelectrical impedance measurements by tetra-polar whole body (TWB, Biodynamic Body Composition Analyser, model 310) and bipolar for upper body (BUB, OMRON $N^{T M}$, model HBF-306BL) after $12 \mathrm{~h}$ fast. Relative body fat (\%BF), lean body mass (LBM) and fat mass (FM) estimation were established from information supplied by two analysers. No significant difference was verified for \%BF (10.9 \pm 4.3 vs. $10.0 \pm 4.2 \% ; P=0.09)$, FM (8.1 \pm 3.5 vs. $7.4 \pm 3.5 \mathrm{~kg} ; P=0.10)$ or $L B M(64.9 \pm 5.2$ vs. $65.5 \pm 4.9 \mathrm{~kg} ; P=0.09)$ between TWB and $B U B$, respectively. Positive significant correlations ( $P<0.001)$ were found between both analysers for LBM $(r=0.948)$, FM $(r=$ $0.891)$ and $\% B F(r=0.859)$. The agreement thresholds were $-0.6 \pm 3.2 \mathrm{~kg}$ for $\operatorname{LBM}(r=0.135 ; P=0.551), 0.8 \pm$ $4.5 \%$ for $\% \mathrm{BF}(r=0.050 ; P=0.824)$, and $0.6 \pm 3.2 \mathrm{~kg}$ for FM $(r=0.032 ; P=0.886)$. The results suggest that the body composition estimation by bioelectrical impedance does not seem to be affected by use of different analysers (BUB vs. TWB) or the total body adiposity.
\end{abstract}

Keywords: lean body mass, body fat, bioelectrical impedance.

\section{INTRODUÇÃO}

Com o avançar dos conhecimentos científicos e tecnológicos na área de estudo da composição corporal, tem sido verificado que os métodos, as técnicas e os procedimentos utilizados para obtenção de informações relativas aos diferentes componentes corporais nem sempre são adequados ou aplicáveis a populações com características distintas ou, até mesmo, não são suficientemente sensíveis para produzir as informações desejáveis com um nível de precisão aceitável.

Assim, no âmbito da pesquisa, a utilização de métodos multicompartimentais que produzem informações mais consistentes sobre os diferentes componentes da composição corporal, a partir da análise de três, quatro ou cinco compartimentos, tem gradativamente recebido a preferência dos pesquisadores, embora a utilização de tais métodos não seja viável para a maioria dos estudos de campo.

Por outro lado, métodos bicompartimentais, como antropometria, espessura de dobras cutâneas e impedância bioelétrica (IB), continuam sendo extensivamente utilizados por pesquisadores e profissionais, sobretudo das áreas de saúde e do esporte, em razão de apresentar boa reprodutibilidade, baixo custo operacional, boa fidedignidade, por ser seguros e não invasivos, e permitir ampla aplicação ${ }^{(1,2)}$. 
O método de IB, especificamente, apresenta uma vantagem adicional a outros métodos bicompartimentais por poder proporcionar informações, dependendo do analisador a ser utilizado, sobre a água corporal total e suas frações intra e extracelular. Além disso, a qualidade das medidas fornecidas pelos analisadores de IB depende muito pouco da habilidade do operador do equipamento, diferente do que ocorre com a antropometria e medidas de espessura de dobras cutâneas, métodos cuja qualidade das informações produzidas é dependente, em grande parte, da experiência do avaliador e de sua qualidade técnica para obtenção das medidas em diferentes pontos anatômicos ${ }^{(3)}$.

Recentemente, tem sido observado aumento no emprego de analisadores de IB bipolares para membros superiores (BMS) ou inferiores, por se tratar de equipamentos de fácil manuseio, relativamente baratos e que fornecem informações rapidamente, sem a necessidade de utilização de eletrodos em locais padronizados ${ }^{(4)}$. Diferente dos analisadores de IB tetrapolares de corpo inteiro (TCI), nos quais a impedância e a água corporal total são estimadas por meio da passagem de uma corrente elétrica entre o pé e a mão direita do avaliado na posição supinada ${ }^{(5)}$, nos analisadores de IB bipolares, a corrente elétrica é transmitida por sensores metálicos que, em contato com as mãos ou pés, registram a impedância de diferentes segmentos corporais entre membros superiores e tronco ou somente entre membros inferiores ${ }^{(1,6,7)}$.

Embora os membros superiores e inferiores, conjuntamente, possam representar cerca de $90 \%$ da impedância total do corpo, os modelos matemáticos utilizados nos aparelhos bipolares assumem que a distribuição da composição corporal é semelhante nos demais segmentos corporais não avaliados ${ }^{(8)}$. Entretanto, as suposições de que a passagem da corrente elétrica entre membros superiores e tronco ou somente entre os membros inferiores reflete a impedância do corpo, como um todo, são questionáveis. Dessa forma, as medidas produzidas acabam sendo suscetíveis de imprecisão ${ }^{(9)}$.

Considerando que as técnicas de medida dos analisadores $\mathrm{TCl}$ e BMS são diferentes, mas que as variáveis a serem medidas (impedância bioelétrica) e estimadas (componentes da composição corporal) são semelhantes, espera-se que as informações produzidas por ambos os equipamentos apresentem boa concordância e que resultem em estimativas relativamente semelhantes dos componentes da composição corporal. Todavia, as informações disponíveis a esse respeito, até o momento, são inconclusivas (3,9-12).

As diferenças encontradas entre os diferentes estudos podem estar atreladas a inúmeros fatores, tais como idade, sexo e gordura corporal total da população utilizada como referência para o modelo matemático desenvolvido pelo fabricante ou, ainda, pela simples aplicação de informações disponibilizadas pelos equipamentos em equações preditivas desenvolvidas para populações específicas, mas não validadas em outras populações.

Nesse sentido, o objetivo deste estudo foi analisar as estimativas de massa livre de gordura (MLG), massa gorda (MG) e gordura corporal relativa (\%GC) e a concordância das medidas produzidas por meio de dois diferentes analisadores de IB (TCl e BMS) em homens adultos jovens.

\section{MÉTODOS}

\section{Sujeitos}

Participaram do estudo 22 universitários do sexo masculino $(23,6 \pm 2,9$ anos; 73,0 $\pm 6,8 \mathrm{~kg} ; 177,6 \pm 6,0 \mathrm{~cm} ; 23,1 \pm 1,9 \mathrm{~kg} / \mathrm{m}^{2}$ ), aparentemente saudáveis. Todos os participantes, após receber informações sobre as finalidades do estudo e os procedimentos aos quais seriam submetidos, assinaram um Termo de Consentimento Livre e Esclarecido. Este estudo faz parte de um projeto longitudinal sobre o efeito de diferentes sistemas de TP sobre variáveis morfológicas, hemodinâmicas e neuromusculares, aprovado pelo Comitê de Ética da Universidade Estadual de Londrina (processo nº 265/06).

\section{Antropometria}

Medidas antropométricas da massa corporal e estatura foram utilizadas para caracterização da amostra. A massa corporal foi mensurada em uma balança de leitura digital, da marca Urano, modelo PS 180A, com precisão de $0,1 \mathrm{~kg}$, ao passo que a estatura foi determinada em um estadiômetro de madeira com precisão de $0,1 \mathrm{~cm}$, de acordo com os procedimentos descritos por Gordon et al..(13). A partir dessas medidas foi calculado o índice de massa corporal (IMC), por meio da relação entre a massa corporal e o quadrado da estatura, sendo a massa corporal expressa em quilogramas (kg) e a estatura em metros (m).

\section{Impedância bioelétrica}

Para a estimativa da composição corporal por IB, os sujeitos compareceram ao laboratório pela manhã, após um jejum de 12h, para a obtenção das medidas por meio de analisadores TCI e BMS.

Ao chegarem ao local, os participantes deitaram-se em decúbito dorsal sobre uma maca isolada de condutores elétricos e se mantiveram nessa posição por cinco a 10 minutos, sem portar qualquer objeto metálico. Na sequência, eletrodos emissores foram colocados sobre a superfície da mão e do pé direito, próximos às articulações das falanges do metacarpo e metatarso, respectivamente. Os eletrodos receptores foram colocados no ponto médio entre as proeminências distais do rádio e da ulna do punho direito, e entre o maléolo medial e lateral do tornozelo direito, respectivamente, como descrito por Lukaski et al.(14). A partir daí, as informações foram coletadas por um analisador TCI (Biodynamic Body Composition Analyser, modelo 310 - Biodynamics Corporation, Seattle, EUA).

A seguir, os participantes foram posicionados em pé, com os cotovelos estendidos e os braços em paralelo, formando um ângulo de 90 graus em relação ao tronco. Com as mãos segurando nos sensores metálicos do analisador BMS (Omron ${ }^{t m}$, modelo HBF-306BL - Omron Healthcare Corporation, Kyoto, Japão), as medidas foram executadas.

Os sujeitos foram orientados, previamente, para seguir rigorosamente as seguintes recomendações, antes da realização das medidas: a) urinar cerca de 30 min antes; b) abster-se da ingestão de alimentos ou bebidas ao longo das últimas quatro horas; c) abster-se do consumo de álcool e bebidas cafeinadas ao longo das últimas 48h; d) evitar esforços físicos vigorosos ao longo das últimas 24h; e) não utilizar diuréticos ao longo dos últimos sete dias ${ }^{(15)}$.

\section{Tratamento estatístico}

Considerando que o teste de Shapiro-Wilk confirmou a normalidade na distribuição dos dados, estatística paramétrica foi utilizada. O teste $t$ de Student para amostras dependentes foi empregado nas comparações entre os dois analisadores de IB investigados. O coeficiente de correlação de Pearson (r) foi utilizado para a análise das possíveis associações entre as variáveis da composição corporal obtidas pelos diferentes analisadores de IB. A análise dos limites de concordância para MLG, MG e \%GC, estimadas por meio dos analisadores BMS e TCl, foi adotada, a partir da plotagem de Bland-Altman ${ }^{(16)}$. O nível de significância estabelecido para todas as análises foi de $P<0,05$. Os dados foram processados no pacote estatístico SPSS, versão 16.0. Para o cálculo do tamanho da amostra foi adotado um alfa de 95\% e um poder de $80 \%$ para os componentes da composição corporal analisados.

\section{RESULTADOS}

Nenhuma diferença estatisticamente significante $(P>0,05)$ foi identificada entre as médias da \%GC, MG e MLG, estimadas pelos analisadores TCl e BMS (Tabela 1). Apesar disso, em valores absolutos, a média da MLG obtida por meio do analisador TCI foi 1,0\% inferior 
à média do BMS. Por outro lado, as médias da MG e \%GC obtidas a partir do analisador $\mathrm{TCl}$, em valores absolutos, foram superiores $(9,5 \% \mathrm{e}$ 0,9 pontos percentuais, respectivamente) àquelas estimadas pelo analisador BMS. Os valores da água corporal total da MLG estimados pelo analisador TCI foram na ordem de 70,9 $\pm 1,3 \%$ (68,1-72,9\%), ao passo que os valores da resistência e da reactância foram, respectivamente, de 450,7 $\pm 47,0 \Omega$ e $66,1 \pm 7,6 \Omega$.

Tabela 1. Comparação entre as estimativas da gordura corporal relativa (\%GC), da massa gorda (MG) e da massa livre de gordura (MLG) a partir das informações fornecidas pelos analisadores tetrapolar de corpo inteiro (TCl) e bipolar para membros superiores (BMS) em homens adultos jovens $(n=22)$.

\begin{tabular}{c|c|c|c}
\hline & TCI & BMS & $P$ \\
\hline$\% G C$ & $10,9 \pm 4,3$ & $10,0 \pm 4,2$ & 0,09 \\
\hline MG (kg) & $8,1 \pm 3,5$ & $7,4 \pm 3,5$ & 0,10 \\
\hline MLG (kg) & $64,9 \pm 5,2$ & $65,5 \pm 4,9$ & 0,09 \\
\hline
\end{tabular}

Nota. Os resultados estão apresentados em valores de média ( \pm DP).

Correlações positivas significantes $(P<0,001)$ e de grande magnitude foram identificadas entre os dois analisadores investigados para $\% G C(r=0,859), M G(r=0,891)$ e $M L G(r=0,948)$ (Figura 1). Os EPE produzidos foram da ordem de 20,6\% para \%GC; $20,2 \%$ para MG e 2,6\% para MLG.

A análise da concordância entre os analisadores TCl e BMS, incluindo o intervalo de confiança a 95\% ( $\pm 1,96$ DP) para as variáveis MLG, MG e \%GC está representada na Figura 2. Para a MLG os limites de concor- dância foram da ordem de -0,6 $\pm 3,2 \mathrm{~kg}$, sem indicativo de tendência na variabilidade individual $(r=0,135 ; P=0,551)$. De forma semelhante, nenhuma tendência foi verificada entre a média dos valores estimados pelos analisadores $\mathrm{TCl}$ e BMS e a diferença das médias, tanto para a MG $(r=0,032 ; P=0,886)$ quanto para a $\% G C(r=0,050 ; P=0,824)$. Vale destacar que os limites de concordância para a \%GC foram mais amplos do que para a $M G(0,8 \pm 4,5 \%$ vs. 0,6 $\pm 3,2 \mathrm{~kg}$, respectivamente).

\section{DISCUSSÃO}

Apesar das diferentes técnicas de medida utilizadas por analisadores TCl e BMS, o presente estudo não verificou diferenças estatisticamente significantes na composição corporal estimada por meio dos dois analisadores investigados ( $\mathrm{TCl}$, Biodynamic Body Composition Analyser, modelo 310 e BMS, Omron ${ }^{T M}$, modelo HBF-306BL). Os resultados encontrados corroboram informações relatadas anteriormente em outros estudos que utilizaram analisadores TCl e BMS, contudo, de marcas e modelos diferentes dos equipamentos utilizados nesta investigação ${ }^{(3,9)}$.

Entretanto, os resultados encontrados devem ser analisados com cautela, uma vez que a ausência de um método de referência neste estudo impossibilita análise mais consistente das estimativas da composição corporal produzidas pelos analisadores TCI e BMS. Assim, o máximo que se poderia afirmar, preliminarmente, é que os dois analisadores parecem produzir estimativas de \%GC, MG e MLG relativamente semelhantes, embora a precisão seja discutível. Partindo do pressuposto de que as informações produzidas se limitam à análise de determinado grupo de sujeitos, seria interessante neste caso, em particular, avaliar o comportamento individual.

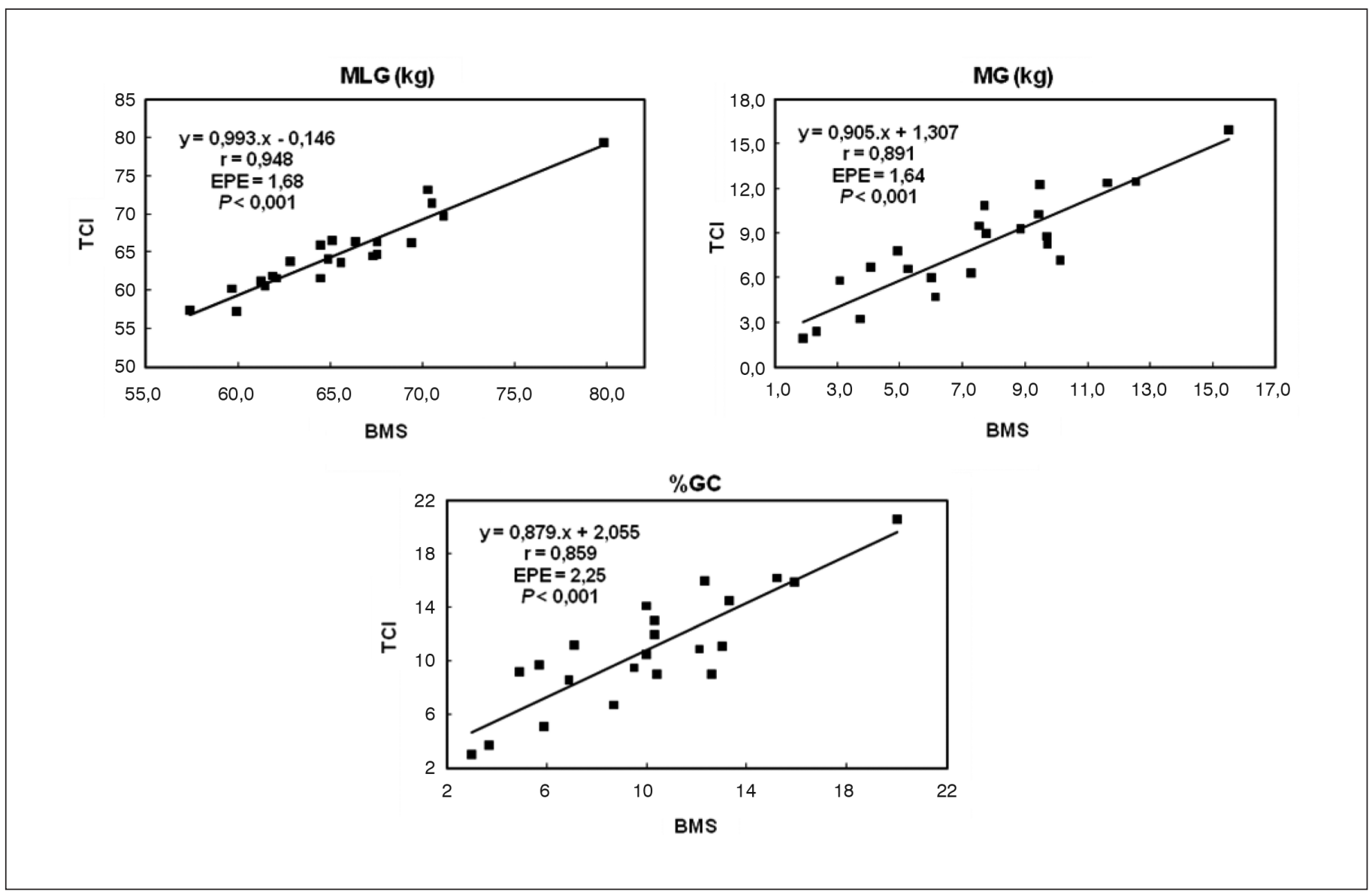

Figura 1. Correlação entre os valores estimados por meio dos analisadores de impedância bioelétrica tetrapolar de corpo inteiro (TCl) e bipolar para membros superiores (BMS) para a massa livre de gordura (MLG), massa gorda (MG) e gordura corporal relativa (\%GC) $(n=22)$. 

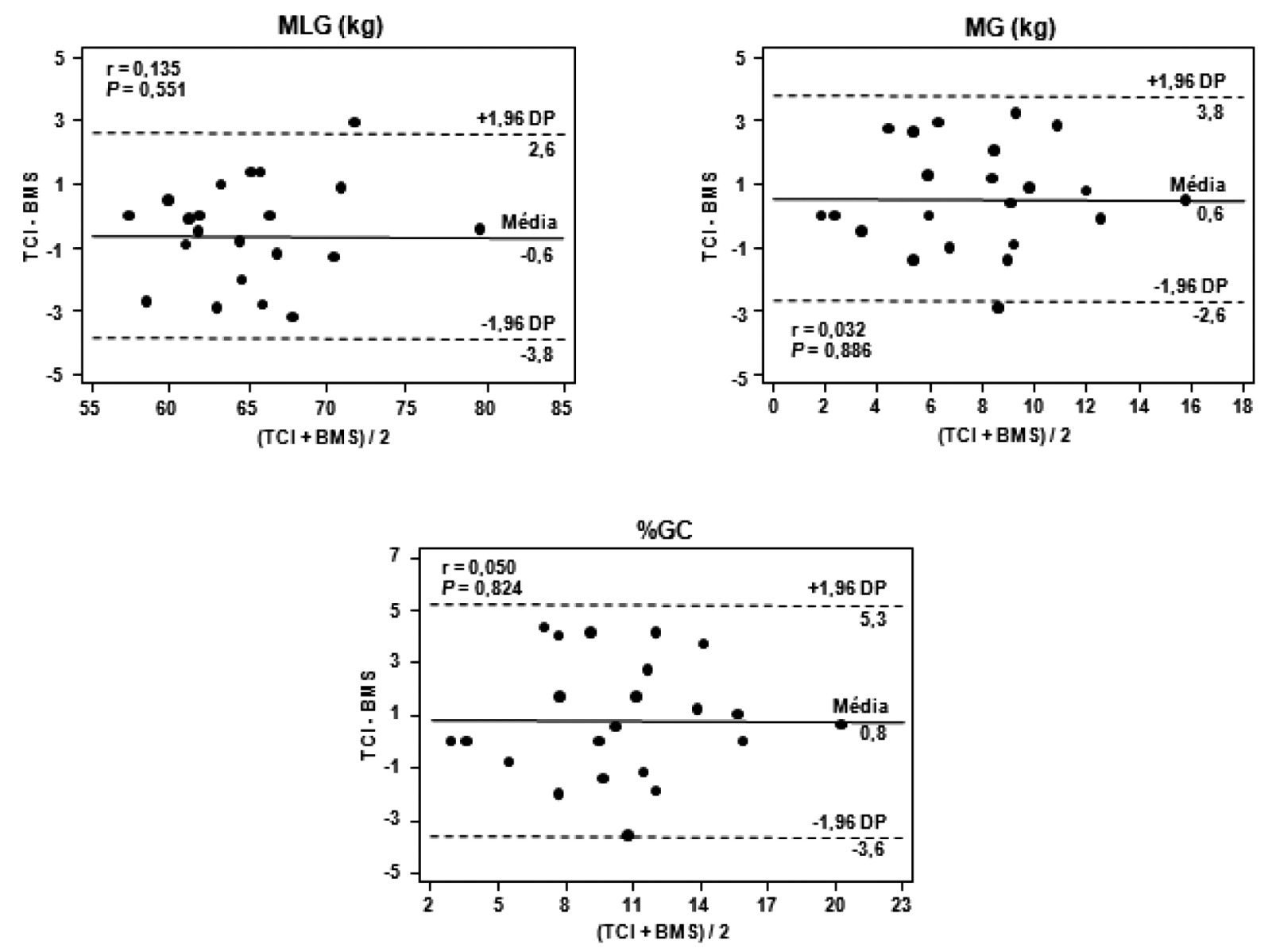

Figura 2. Limites de concordância entre os valores estimados por meio dos analisadores de impedância bioelétrica bipolar para membros superiores (BMS) e tetrapolar de corpo inteiro (TCl) para a massa livre de gordura (MLG), massa gorda (MG) e gordura corporal relativa (\%GC) $(n=22)$. Um ponto está sobreposto na variável MLG.

Nesse sentido, a análise de concordância utilizada entre TCI e BMS indicou limites relativamente amplos para as variáveis analisadas, confirmando achados publicados anteriormente ${ }^{(9)}$. Desse modo, a MLG poderia ser subestimada em até $2,6 \mathrm{~kg}$ ou superestimada em até $3,8 \mathrm{~kg}$, a \%GC poderia ser subestimada em até $5,3 \%$ ou superestimada em até $3,7 \%$ e a MG poderia ser subestimada em até 3,8kg ou superestimada em até 2,6kg pela técnica BMS em face de TCI. Todavia, nenhuma associação entre as diferenças das técnicas TCl e BMS e a médias das diferenças foi identificada.

Considerando que os maiores valores individuais da \%GC foram estimados pelo analisador $\mathrm{TCl}$ em 16 sujeitos ( $73 \%$ da amostra estudada), os resultados encontrados, aparentemente, iriam de encontro àqueles relatados por Dittmar ${ }^{(9)}$. Entretanto, ao se analisar apenas os resultados dos sujeitos que apresentavam valores de adiposidade corporal semelhantes aos da amostra do presente estudo, as informações tornam-se similares. Esse fato parece indicar que o analisador BMS tende a subestimar os valores de \%GC em homens magros (< 15\%) quando comparado com analisador TCl.

Um aspecto importante a ser destacado é que as análises da composição corporal neste estudo foram estabelecidas com base nas informações apresentadas por cada analisador, especificamente, de acordo com os modelos matemáticos definidos pelos próprios fabricantes, visto que essa é uma situação bastante frequente entre os usuários desses equipamentos.

Entretanto, esse fato tem merecido severas críticas de especialistas em estudos da composição corporal, uma vez que existem evidências suficientes na literatura de que a margem de erro nas estimativas da composição corporal, principalmente por meio de modelos bicompartimentais (MG e MLG), pode ser comprometida sobremaneira pelo uso indiscriminado de modelos matemáticos desenvolvidos e validados em determinadas populações, em sujeitos com características distintas da população que deu origem à equação preditiva escolhida(2).

Assim, pesquisadores têm desenvolvido e validado equações preditivas para o método de IB, em diferentes populações ${ }^{(3,17,18)}$, na tentativa de melhorar as estimativas da composição corporal produzidas por esse método frente aos métodos considerados de referência (pesagem hidrostática, absortometria radiológica de dupla energia e modelos multicompartimentais). Esse fato é plenamente justificável, uma vez que algumas variáveis, tais como o sexo, a idade, a etnia, o nível de aptidão física individual, o estado de hidratação, entre outras, podem afetar, sobretudo, a densidade dos tecidos magros.

Tomando como exemplo os dados originais da presente investigação, se aplicássemos as informações fornecidas pelo equipamento TCI na equação preditiva proposta por Segal et al.(17) os valores médios ( \pm DP) da \%GC para amostra investigada seriam da ordem de 12,6 \pm 1,9. Utilizando essa informação para a determinação da MLG e MG e, posteriormente, comparando os valores encontrados com as estimativas geradas pelo analisador BMS, chegaríamos à conclusão de que o analisador BMS teria subestimado significantemente $(P<0,001)$ a \%GC e, consequentemente, a MG, e superestimado a MLG.

Além disso, a aplicação dos procedimentos propostos por Bland \& Altman ${ }^{(16)}$ seria fundamental para a análise dos dados, uma vez que, 
apesar de os valores do coeficiente de correlação de Pearson, tal como foi verificado no presente estudo, indicarem a existência de boa correlação entre os valores da composição corporal estimados pelos dois analisadores $(r=0,94$ para MLG; $r=0,92$ para MG; e $r=0,88$ para \%GC; $P<0,01)$, não haveria boa concordância entre as medidas obtidas individualmente. Resumindo, os resultados da análise de concordância entre os dois analisadores nos indicaria que o analisador BMS, quando comparado com o analisador $\mathrm{TCl}$ (representado pela equação citada anteriormente), teria suas estimativas afetadas pela adiposidade corporal total ( $r=-0,83$ e -0,87 para MG e \%GC, respectivamente; $P<0,0001$ ), apresentando pouca sensibilidade para discriminar valores extremos, subestimando os valores de gordura corporal relativa nos sujeitos muito magros e superestimando naqueles com excesso de gordura. Essas informações confirmam as limitações da utilização da análise de correlação, de forma isolada, para testar a validade de determinado método frente a um método adotado como referência, ou seja, a força da correlação não indica concordância entre dois métodos ${ }^{(19)}$.

O exemplo apresentado anteriormente demonstra como o maior ou menor rigor metodológico na avaliação da composição corporal pode influenciar os resultados de pesquisas ou, ainda, interferir na conduta profissional. Os resultados conflitantes indicam que o uso indiscriminado dos analisadores investigados pode induzir uma interpretação equivocada da composição corporal, comprometendo a tomada de decisão, sobretudo, em situações de acompanhamento.

Portanto, as informações encontradas neste estudo parecem válidas quando os equipamentos são comparados entre si, a partir dos algoritmos definidos pelos próprios fabricantes. Por outro lado, vieses de interpretação podem ser gerados quando outras equações preditivas são utilizadas nas comparações, até mesmo quando são empregadas informações disponibilizadas pelo próprio equipamento, como o valor da resistência ou reactância ${ }^{(17)}$.
Embora o presente estudo apresente achados interessantes, a ausência de um método de referência para avaliação da composição corporal não permite uma análise mais consistente sobre a real precisão dos valores encontrados para as diferentes variáveis avaliadas. Apesar disso, os resultados relatados em estudos de validação com analisadores TCI nos levam a acreditar que as estimativas da composição corporal por essa técnica sejam válidas e confiáveis ${ }^{(14)}$. Todavia, novos estudos nessa direção, confrontando analisadores TCl e BMS com métodos de referência, em diferentes populações, ainda, se fazem necessários.

\section{CONCLUSÃO}

Os resultados deste estudo sugerem que as estimativas da composição corporal não parecem ser influenciadas pelo uso de diferentes analisadores de IB (BMS e TCI), mas sim pelo uso indiscriminado de equações preditivas que adotam como variáveis independentes informações fornecidas pelos equipamentos.

Adicionalmente, a análise de concordância entre os equipamentos para \%GC, MG e MLG revelou que as estimativas geradas pelos analisadores TCl e BMS não tendem a ser afetadas pela adiposidade corporal total dos sujeitos avaliados.

\section{AGRADECIMENTOS}

Os autores agradecem a CAPES, CNPq e FAPESP pelas bolsas outorgadas e aos estudantes de pós-graduação Ademar Avelar de Almeida Júnior e Renata Selvatici Borges Januário pela contribuição para a execução do experimento.

Todos os autores declararam não haver qualquer potencial conflito de interesses referente a este artigo.

\section{REFERÊNCIAS BIBLIOGRÁFICAS}

1. Deurenberg P, Deurenberg-Yap M. Validation of skinfold thickness and hand-hand impedance measurements for estimation of body fat percentage among Singaporean Chinese, Malay and Indian subjects. Asia Pac J Clin Nutr. 2002;11:1-7.

2. Heymsfield SB, Wang Z, Visser M, Gallagher D, Pierson Jr RN. Techniques used in the measurement of body composition: an overview with emphasis on bioelectrical impedance analysis. Am J Clin Nutr. 1996;64:4785-484S.

3. Demura S, Yamaji S, Goshi F, Kobayashi H, Sato S, Nagasawa Y. The validity and reliability of relative body fat estimates and the construction of new prediction equations for young Japanese adult males J Sports Sci. 2002;20:153-64.

4. Gibson AL, Heyward VH, Mermier CM. Predictive accuracy of Omrom body logic analyzer in estimating relative body fat of adults. Int J Sport Nutr Exerc Metab. 2000;10:216-27.

5. Lukaski HC, Siders WA. Validity and accuracy of regional bioelectrical impedance devices to determine whole-body fatness. Nutrition 2003;19:851-7.

6. Biggs J, Cha K, Horch K. Electrical resistivity of the upper arm and leg yields good estimates of whole body fat. Physiol Meas. 2001;22:365-76.

7. Miyatani M, Kanehisa H, Masuo Y, Ito M, Fukunaga T. Validity of estimating limb muscle volume by bioelectrical impedance. J Appl Physiol. 2001;91:386-94

8. Heitman BL. Impedance a valid method in assessment of body composition? Eur J Clin Nutr. 1994;48:228-40.

9. Dittmar M. Comparison of bipolar and tetrapolar impedance techniques for assessing fat mass. Am J Hum Biol. 2004;16:593-7.
10. Cox-Riejven PLM, Kreel BV, Soeters PB. Bio-electrical impedance spectroscopy: alternatives for the conventional hand-to-foot measurements. Clin Nutr. 2002;21:127-33

11. Leite CMBA, Mulinari RA, Carvalho JGR. Estimativa da gordura corporal através da bioimpedância tetrapolar: estudo comparativo entre o método convencional e um monitor de gordura corporal. Rev Bras Nutr Clin. 2003;18:26-30.

12. Lintsi M, Kaarma H, Kull I. Comparison of hand-to-hand bioimpedance and anthropometry equations versus dual-energy $\mathrm{X}$-ray absorptiometry for the assessment of body fat percentage in 17-18-year-old conscripts. Clin Physiol Funct Imaging. 2004;4:85-90.

13. Gordon CC, Chumlea WC, Roche AF. Stature, recumbent length, and weight. In: Lohman TG, Roche AF, Martorell R, editors. Anthropometric standardization reference manual. Champaign: Human Kinetics, 1988;3-8.

14. Lukaski HC, BolonchukWW, Hall CB, Siders WA. Validation of tetrapolar bioelectrical impedance method to assess human body composition. J Appl Physiol. 1986;60:1327-32

15. Heyward VH. ASEP methods recommendation: body composition assessment. JEP. 2001;4:1-12.

16. Bland JM, Altman DG. Statistical methods for assessing agreement between two methods for clinical measurement. Lancet. 1986;8:307-10.

17. Segal KR, Van Loan M, Fitzgerald PI, Hodgdon JA, Van Itallie TB. Lean body mass estimation by bioelectrical impedance analysis: a four-site cross-validation study. Am J Clin Nutr. 1988:47:7-14.

18. Lockner DW, Heyward VH, Griffin SE, Marques MB, Stolarczyk LM, Wagner DR. Cross-validation of modified fatness-specific bioelectrical impedance equations. Int I Sport Nutr. 1999;9:48-59.

19. Williams CA, Bale P. Bias and limits of agreement between hydrodensitometry, bioelectrical impedance and skinfold calipers measures of percentage body fat. Eur J Appl Physiol. 1998;77:271-7. 